\title{
2. Überblick zu aktuellen Forschungsbefunden
}

\subsection{Geräteausstattung und Gerätenutzung}

Die Mediennutzung junger Menschen hat sich in den letzten Jahrzehnten entscheidend gewandelt. Am deutlichsten zeigt sich dies in der medialen Ausstattung. Mittlerweile besitzen 42 Prozent (Ost: 51 \%; West: 38 \%) der 6- bis 13-jährigen Kinder und Jugendlichen ein eigenes Fernsehgerät, 35 Prozent (Ost: $51 \%$; West: 33 \%) eine eigene Spielkonsole und 12 Prozent (Ost: $20 \%$; West: $11 \%$ ) einen eigenen Computer in ihrem Zimmer (Feierabend \& Rathgeb, 2006b). Unter Jugendlichen ab 14 Jahren sind Fernsehbesitzer mit knapp 70 Prozent bereits in der Mehrheit, eine Quote die Mitte der achtziger Jahre bundesweit noch bei 29 Prozent lag (Feierabend \& Klingler, 2001). Jungen weisen durchweg eine höhere Ausstattung mit Mediengeräten auf als Mädchen, wobei sich besonders große Unterschiede beim Besitz von Computer und Spielkonsole zeigen (Feierabend \& Rathgeb, 2006a, 2006b). So haben derzeit 64 Prozent der 12- bis 19-jährigen Jungen einen eigenen Fernseher (Mädchen: $58 \%$ ), 65 Prozent einen eigenen Computer (Mädchen: $48 \%$ ) und 52 Prozent eine eigene Spielkonsole (Mädchen: $20 \%$ ) in ihrem Zimmer (Feierabend \& Rathgeb, 2006a).

Eine höhere Ausstattung mit Mediengeräten ist mit höheren Nutzungszeiten verbunden. So zeigt sich, dass der Besitz eines eigenen TV-Geräts im Zimmer sowohl mit einer rein zeitlichen (Kinder mit eigenem TV-Gerät schauen im Durchschnitt pro Tag 80 Minuten länger fern, KFN-Befragung 2003) als auch mit einer inhaltlichen Ausweitung der Fernsehnutzung einhergeht. Längsschnittuntersuchungen zeigen, dass der Konsum von Gewalt- und Horrorfilmen unter Heranwachsenden in den neunziger Jahren weiter stark angestiegen ist (z. B. Fuchs, M., Lamnek \& Luedtke, 2001; Fuchs, M., Lamnek, Luedtke \& Baur, 2005). Ferner ist das Fernsehen unter den 6- bis 13-Jährigen mit 97 Prozent der Nennungen die Freizeitaktivität, die am häufigsten mindestens einmal pro Woche ausgeübt wird, gefolgt vom Hausaufgaben machen/Lernen und Freunde treffen mit 96 Prozent (Feierabend \& Rathgeb, 2006b). Videos bzw. DVDs werden von 60 Prozent der Kinder mindestens einmal pro Woche geguckt (Feierabend \& Rathgeb, 2006b).

Im Bereich der Computerspiele ${ }^{1}$ hat sich in einer Befragung von Eltern $(n=245)$ mit Klein- und Vorschulkindern (2 - 5 Jahre) gezeigt, dass zwar in einem hohen Anteil der untersuchten Haushalte die nötige Spielehardware zur Verfügung steht (65\% Computer, $31 \%$ Spielkonsole), sich jedoch nur 3 Prozent der Klein- und Vor-

1 Im vorliegenden Dokument wird für die Begriffe Computerspiel und Videospiel pauschal die Bezeichnung „Computerspiel“" verwendet, solange nicht auf exklusive, plattformspezifische Merkmale von Computer- oder Videospielen Bezug genommen wird. 
schulkinder ein- oder mehrmals in der Woche mit dem Computer beschäftigen, 11 Prozent nutzen ihn selten (Feierabend \& Mohr, 2004). Mit höherem Alter steigt die Nutzungsintensität jedoch sehr deutlich an (Feierabend \& Klingler, 2003b; Feierabend \& Rathgeb, 2006b; Wake, Hesketh \& Waters, 2003). Wie die vom Medienpädagogischen Forschungsverbund Südwest herausgegebene KIM-Studie zeigt (Feierabend \& Klingler, 2003b; Feierabend \& Rathgeb, 2006b), benutzten im Jahre 2005 bereits 76 Prozent aller 6- bis 13-jährigen Kinder zumindest hin und wieder einen Computer, 56 Prozent nutzten ihn ein- bis mehrmals in der Woche und 26 Prozent fast jeden Tag. Das Spielen von Computerspielen ist hierbei die beliebteste Tätigkeit: 63 Prozent der PC nutzenden Kinder spielt mindestens einmal wöchentlich alleine, 50 Prozent gemeinsam mit anderen Computerspiele (Feierabend \& Rathgeb, 2006b). Zusätzlich nutzten 44 Prozent der Kinder mindestens einmal in der Woche eine tragbare Spielkonsole (z. B. Gameboy) und 40 Prozent eine stationäre Spielkonsole (Feierabend \& Klingler, 2003b). Mädchen spielen jedoch deutlich weniger als Jungen (Feierabend \& Klingler, 2003b; Feierabend \& Rathgeb, 2006b; Funk, 1993; Gentile, Lynch, Linder \& Walsh, 2004; Griffiths \& Hunt, 1998; van Schie \& Wiegman, 1997; Wake et al., 2003). ${ }^{2}$

Seit Verbreitung des Fernsehens beginnend in den 1950er Jahren sowie von Computerspielen in den 1980er Jahren wurden international zahlreiche Studien zum Einfluss dieser Medien auf kognitive und schulische Leistungen von Kindern und Jugendlichen durchgeführt. Negative und positive Effekte wurden und werden hierbei zum Teil kontrovers diskutiert. Derzeit weist die Forschungslage jedoch mehrheitlich auf einen negativen Zusammenhang zwischen exzessiver Mediennutzung und Schulleistung hin. Im Folgenden wollen wir einen Überblick über relevante Studien der letzten Jahre geben, die sich mit dem Zusammenhang von Mediennutzung und Schulleistung beschäftigt haben.

\subsection{Wirkungsforschung Fernsehen und Schulleistung}

Bereits für Kleinkinder zeigen sich sehr bedenkliche Befunde: So gibt eine Längsschnittanalyse (Christakis, Zimmerman, DiGiuseppe \& McCarty, 2004), die in den USA mit über 1.000 Kleinkindern durchgeführt wurde, Hinweise auf einen Zusammenhang zwischen frühem Fernsehen (mit 1 bis 3 Jahren) und späterem Auftreten einer Aufmerksamkeitsstörung (ADHD) im Alter von 7 Jahren. Im Alter von einem

2 Der Umsatz, der mit Video- und Computerspielen weltweit Jahr für Jahr erzielt wird, überstieg 2004 mit ca. 18,8 Mrd. Euro sogar den Umsatz von Kinofilmproduktionen. Das Marktforschungsinstitut „Research And Markets“ sagt voraus, dass bis zum Jahre 2008 die weltweiten Jahreseinnahmen auf 27,1 Mrd. Euro ansteigen werden (VUD, 2005). Deutschland liegt dabei zwar deutlich hinter den USA, stellt aber in Europa hinter Großbritannien den zweitstärksten Unterhaltungssoftwaremarkt dar. 2004 wurden hier 1.3 Mrd. Euro Marktwert an Video- und Computerspielen umgesetzt (VUD, 2005). 
Jahr schauten die untersuchten Kinder im Durchschnitt bereits 2,2 Stunden $(S D=2,91)$ täglich fern, im Alter von 3 Jahren 3,6 Stunden $(S D=2,94)$. Bei Steigerung der Fernsehzeit im ersten Lebensjahr um eine Standardabweichung (zwei bis drei Stunden) erhöht sich das Risiko von ADHD im Alter von 7 Jahren um 28 Prozent. Zusammenhänge zwischen einer Erkrankung an ADHD und Schulleistung sind wiederum als hoch relevant einzuschätzen, da sich diese Störung vor allem durch Symptome der Unaufmerksamkeit, wie z. B. Flüchtigkeitsfehler, mangelndes Zuhören, fehlende Aufgabenerledigung und leichte Ablenkbarkeit etc. kennzeichnet (Comer \& Sartory, 2001). So zeigte sich in einer weiteren Längsschnittstudie an 103 Jungen mit ADHD und 100 Jungen ohne eine solche Erkrankung unter anderem, dass die erkrankten Jungen zu 25 Prozent die Highschool abbrechen und nur zu 12 Prozent das College abschließen. Die ,gesunden“ Jungen hingegen brachen die Highschool nur in 2 Prozent der Fälle ab und schlossen zu 50 Prozent das College ab (Mannuza et al., 1993, zitiert nach Comer \& Sartory, 2001).

Andere Studien belegen unmittelbare negative Effekte zwischen Fernsehkonsum und der Rechen-, Sprach- und Lesekompetenz von Kindern. In einer Untersuchung mit fast 1.800 US-amerikanischen Kindern (Zimmerman \& Christakis, 2005) wurden die Rechen- und Leseleistungen im Alter von 6 bzw. 7 Jahren mit dem Fernsehkonsum vor dem dritten sowie zwischen dem dritten und fünften Lebensjahr in Beziehung gesetzt. Laut dieser Studie sitzen Kinder unter 3 Jahren im Durchschnitt bereits über 2 Stunden täglich vor dem Fernseher. Kinder im Alter von 3 bis 5 Jahren schauen über 3 Stunden fern. Jede zusätzliche Stunde, die unter 3-jährige Kinder täglich vor dem Fernseher verbringen, wirkt sich dabei negativ auf ihre Rechen- und Leseleistungen aus, unabhängig vom sozialen Hintergrund des Elternhauses sowie den kognitiven Fähigkeiten der Kinder.

Des Weiteren konnte in einer deutschen Untersuchung zum Zusammenhang von Fernsehkonsum und der Entwicklung der Sprach- und Lesekompetenzen im frühen Grundschulalter mit 332 Kindern (Ennemoser, 2003; Ennemoser, Schiffer, Reinsch \& Schneider, 2003; Schiffer, Ennemoser \& Schneider, 2002) gezeigt werden, dass ein erhöhter Fernsehkonsum sowohl quer- als auch längsschnittlich mit schwächeren Sprach- und Lesekompetenzen einhergeht. Die Untersuchungsstichprobe setzte sich aus zwei Kohorten zusammen, wobei die jüngeren Kinder $(n=165)$, im Durchschnitt 6 Jahre und 4 Monate alt, zu Beginn der Untersuchung im Jahr 1998 im letzten Kindergartenjahr waren; die älteren Kinder $(n=167)$, im Durchschnitt 8 Jahre und 6 Monate alt, befanden sich zu diesem Zeitpunkt in der zweiten Klasse. Die Studie berücksichtigte bis zum Sommer 2003 insgesamt sechs Messzeitpunkte. Sprach- und Leseleistung (Wortschatz, Lesegeschwindigkeit, Leseverständnis, Rechtschreibleistung) sowie Intelligenz wurden mittels psychologischer Testdiagnostik erhoben; der Fernsehkonsum anhand eines Tagebuchs, in welches bei den ersten fünf Messzeitpunkten die Eltern für den Zeitraum von einer Woche den genauen Tagesablauf ihrer Kinder eintrugen. Zum letzten Messzeitpunkt füllten die Kinder das Tagebuch selbst aus. Aufgrund der Daten zum Fernsehkonsum wurden 
die Kinder in drei Gruppen eingeteilt: Wenigseher, mit etwa 15 bis 20 Minuten, Normalseher, mit ca. einer Stunde und Vielseher, mit mehr als 2 Stunden täglichem Fernsehkonsum. ${ }^{3}$ Es zeigte sich, dass eine höhere Fernsehdauer im Kindergarten eine geringere Lesekompetenz in der ersten Klasse vorhersagt. Die Fernsehdauer in der ersten Klasse bedingt wiederum die Lesekompetenz in der dritten Klasse. Lesekompetenz und Fernsehdauer stellen dabei relativ stabile Merkmale dar (vgl. Abbildung 1).

Abbildung 1: Strukturgleichungsmodell zur Analyse kausaler Beziehungen zwischen Fernsehdauer und Lesekompetenz (Ennemoser, 2003, S. 448)

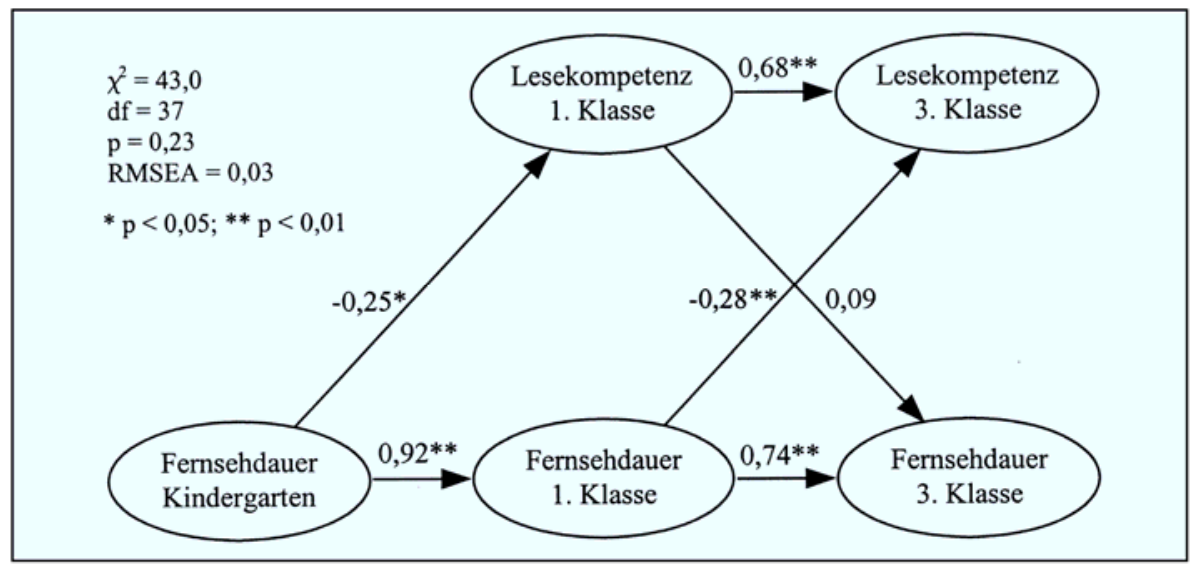

Für eine differenziertere Analyse wurden auch der sozioökonomische Status und das Intelligenzniveau der Kinder berücksichtigt. Neben allgemein schwächerer Sprachund Lesekompetenz bei erhöhtem Fernsehkonsum wird dieser negative Effekt des Vielsehens in der Gruppe der Kinder mit hohem sozialem Status besonders deutlich (Ennemoser et al., 2003). Des Weiteren erbrachten in der jüngeren Altersgruppe (zu Beginn der Untersuchung im letzten Kindergartenjahr) die Vielseher gerade in der Gruppe der weniger intelligenten Kinder besonders schlechte Leistungen in der Sprach- und Lesekompetenz verglichen mit den Wenigsehern (Schiffer et al., 2002).

Die neuseeländischen Forscher Hancox, Milne und Poulton (2005) begleiteten eine Geburtenkohorte von ca. 1.000 zu Beginn 5-jährigen Kindern bis in das Erwachsenenalter (26 Jahre). Das Fernsehverhalten wurde im Alter von 5, 7, 9, 11, 13 und 15 Jahren erhoben und stieg von durchschnittlich 2,06 Stunden in der Kindheit auf 3,13 Stunden in der Adoleszenz an. Als Kovariaten zur abhängigen Variable Bildungsabschluss wurden der Intelligenzquotient (IQ) und der sozioökonomische StaWenigsehern zugeordnet, die bei allen Messzeitpunkten im oberen oder unteren Drittel der Altersgruppe lagen. Die übrigen Kinder, die einen durchschnittlichen Fernsehkonsum aufwiesen, wurden als Normalseher bezeichnet 
tus des Kindes sowie eine Einschätzung möglicher antisozialer und hyperaktiver Verhaltensprobleme des Kindes im Alter von 5 Jahren als Lehrer- und Elternurteile miteinbezogen. Ein hoher IQ sowie ein hoher sozioökonomischer Status des Kindes waren mit dem späteren Erwerb eines besseren Bildungsabschluss verbunden, d. h. in dieser Untersuchungsgruppe gab es im Vergleich zu den anderen Gruppen mehr Erwachsene mit Bildungsabschluss und weniger ohne $(p \leq .001)$. Durch Lehrer und Eltern eingeschätzte Verhaltensprobleme im Alter von 5 Jahren waren hingegen ein Prädiktor für einen geringeren Bildungsabschluss $(p \leq .001)$.

Erhöhte Fernsehzeiten in Kindheit und Jugend standen in unmittelbarem Zusammenhang mit einem niedrigen kindlichen sozioökonomischen Status $(p<.001)$, einem niedrigen IQ $(p<.001)$ und mehr Verhaltensproblemen im Alter von 5 Jahren $(p<.05)$. Bei der Betrachtung des Zusammenhangs von Fernsehnutzungszeiten und Bildungsabschluss zeigte sich, dass sich ein erhöhter Fernsehkonsum im Alter von 5 bis 15 Jahren negativ auf die Realisierung eines Schul- oder Universitätsabschlusses auswirkt. Erwachsene, die als Kinder mehr als 3 Stunden pro Tag vor dem Fernseher verbrachten, hatten mit 26 Jahren öfter keinen Schulabschluss; die mit den niedrigsten Fernsehzeiten hatten am häufigsten einen Universitätsabschluss. Hohe tägliche Fernsehzeiten hingen zwar unmittelbar mit einem niedrigem sozioökonomischen Status der Eltern und einem niedrigem Intelligenzniveau der Kinder zusammen, die Befunde blieben jedoch auch bei Berücksichtigung dieser Faktoren bestehen.

Bei einer differenzierteren Analyse zeigte sich, dass die mittleren Fernsehzeiten (an einem Wochentag) im Alter von 13 bis 15 Jahren ein besonders guter Prädiktor für einen Schulabbruch waren, niedrigere Fernsehzeiten im Alter von 5 bis 11 Jahren hingegen das Erlangen eines Universitätsabschlusses gut vorhersagen konnten. Bei einer Aufteilung der Kohorte in drei IQ-Gruppen (niedrig, mittel, hoch) waren die stärksten Effekte der Fernsehnutzung auf den Universitätsabschluss in der Gruppe mit mittlerem IQ zu beobachten. Hochbegabte (hoher IQ) und weniger Begabte (niedriger IQ) waren in geringerem Maße durch eine hohe Fernsehnutzung beeinträchtigt. Hochbegabte mit höherer Fernsehnutzung erlangten z. B. immer noch häufiger einen Universitätsabschluss.

Neben den Nutzungszeiten kann bereits die Geräteausstattung der Kinder in ihrem Kinderzimmer von großem Einfluss sein. So hat sich gezeigt, dass auch der Besitz eines eigenen Fernsehers im Kinderzimmer vermittelt über längere Nutzungszeiten mit schlechteren Schulleistungen einhergeht. In einer Befragung 386 kalifornischer Drittklässler (Borzekowski \& Robinson, 2005) schauten die Kinder mit einem eigenen Fernseher im Kinderzimmer (immerhin 71 \% der Kinder!) wöchentlich ca. zwei Stunden mehr Fernsehen als Kinder ohne eigenes Fernsehgerät und hatten ferner um 8 bis 10 Prozent niedrigere Schulleistungen beim Rechnen (standardisierte Punktwerte: 53.3 vs. 63.1, $p$.001) und Lesen (standardisierte Punktwerte: 47.5 vs. $55.0, p<.001)$. 
Wenngleich die Forschungslage zu Computerspielen noch weniger differenziert und weit vorangeschritten ist, vermitteln die vorhandenen Studien ein ähnliches Bild. So geht erhöhte Computerspielnutzung den bestehenden Studien zufolge mit einem erhöhten Risiko für schulische Leistungseinbußen einher. Dies wird bereits Mitte der 1980er Jahre berichtet (Harris \& Williams, 1985; Lin \& Lepper, 1987). Da sich jedoch seit den 1980er Jahren der Computerspielmarkt und die Leistungsfähigkeit der Plattformen und Spiele stark verändert haben, müssen neuere Studien herangezogen werden, um die gegenwärtige Relevanz dieser Freizeitbeschäftigung für schulisches Lernen aufzeigen zu können.

Walsh (2000) legt in einer Stellungnahme für den US-Senat vom 21. März 2001 dar, dass sowohl Nutzungszeiten als auch Nutzungsinhalte von Bedeutung für schulische Leistungen sind: Jugendliche, die mehr spielen, geben für sich schlechtere Schulleistungen an. Ebenso schneiden auch Jugendliche, die sich vermehrt Gewaltinhalten aussetzen, in der Schule schlechter ab. Gentile et al. (2004) führten eine Untersuchung an 607 durchschnittlich 14-jährigen Acht- $(\mathrm{n}=496)$ und Neuntklässlern $(\mathrm{n}=111)$ durch. Die Schülerinnen und Schüler wurden nach ihrer Nutzungsdauer von Computerspielen an Wochenenden und normalen Wochentagen befragt, woraus eine wöchentliche Nutzungszeit errechnet wurde. Zusätzlich gaben die Teilnehmer für ihre drei Lieblingsspiele die Nutzungshäufigkeit an und bewerteten deren Gewaltgehalt, jeweils auf einer siebenstufigen Ratingskala. Aus diesen Angaben errechneten die Autoren einen Gewaltexpositionsindex ${ }^{4}$ für jeden Befragungsteilnehmer. Des Weiteren wurden die Schulnoten in unterschiedlichen Schulfächern abgefragt (im amerikanischen Schulsystem von A+ bis F). Im Rahmen der Auswertung wurden diese so umkodiert, dass höhere Werte für bessere Noten stehen. Der negative Zusammenhang zwischen Mediennutzung und Schulleistung bestätigte sich darin, dass sowohl die wöchentlichen Nutzungszeiten $(r=-.25)$ als auch die Computerspielgewalt ermittelt über den Gewaltexpositionsindex $(r=-.23)$ negativ mit Schulleistungen in Form von Schulnoten korrelieren. Jedoch ist der Einfluss von Gewaltexposition auf die Schulnoten statistisch nicht mehr bedeutsam, wenn der Zusammenhang nach allgemeinen Spielzeiten hin kontrolliert wird. Die Autoren sehen hierin eine Bestätigung der Annahme, dass die beeinträchtigende Wirkung des interaktiven Mediums vorrangig durch die zeitliche Beanspruchung verursacht ist (siehe Kapitel 3.1: Zeitverdrängungshypothese)

Der negative Zusammenhang zwischen Computerspielnutzung und Schulleistungen wird auch für ältere Populationen berichtet. In einer Querschnittsuntersuchung von Anderson und Dill (2000) befragten die Autoren 227 Psychologiestudenten und -studentinnen früher Semester (78 männlich, 149 weiblich, 18,5 Jahre Durchschnittsalter) zu ihrem Mediennutzungsverhalten und verglichen ihre Angaben mit sen drei Werten der Mittelwert gebildet. 
ihren Leistungen in der Universität laut Zentralregister. Hier zeigten sich negative Zusammenhänge zwischen Computerspielnutzungszeit und Studienleistungen in Form von Prüfungsnoten $(r=.20)$.

Von besonderer Relevanz sind auch die Ergebnisse einer aktuellen USamerikanischen Repräsentativbefragung, die von Roberts, Foehr und Rideout (2005) im Auftrag der Kaiser Family Foundation an 2.023 Schülern der dritten bis zwölften Klasse (8- bis 18-jährige Kinder und Jugendliche) durchgeführt wurde. Insbesondere Variablen der Medienausstattung und des Mediennutzungsverhaltens wurden umfassend einbezogen. Dies beinhaltet neben Fernsehen, Video/DVD und Computerspielen auch Zeitungen, Zeitschriften, Bücher, Musik, Internet- und Computernutzung. Die Befragungsteilnehmer wurden gebeten, die Nutzungszeiten für den jeweils vorangegangenen Tag in Minuten bzw. Stunden anzugeben. Bei 694 der Befragten kam zusätzlich ein 7-tägiges Mediennutzungstagebuch zum Einsatz. Um die relative Relevanz nichtmedialer Aktivitäten abzubilden, wurde auch die mit Freunden, Eltern, Hausaufgaben, Sport, Haushaltsarbeiten, Jobben und sonstigen Aktivitäten verbrachte Zeit erhoben. Zusätzlich wurden Medieninhalte, der soziale Rezeptionskontext, soziodemografische und sozialpsychologische Daten von den Teilnehmern erfasst (Die Befragungen wurden im Klassenverbund durchgeführt und nahmen ca. 40 Minuten in Anspruch). Den Autoren liegen Vergleichsdaten zu 1999 vor, so dass auch allgemeine Veränderungen in der Medienausstattung und -nutzung betrachtet werden können.

Die Ergebnisse verdeutlichen zunächst erneut die Ausstattungsunterschiede zwischen Jungen und Mädchen: In der aktuellen Befragung geben 72 Prozent der Jungen und 64 Prozent der Mädchen an, einen eigenen Fernseher im Zimmer zu haben. Die Zahlen zeigen, dass die Kinder hinsichtlich eigener Fernsehgeräte bereits mit 8 10 Jahren ein Ausstattungsmaximum erreicht haben, das auch in höherem Lebensalter kaum noch zunimmt. Bei der Videospielkonsole gibt es noch deutlichere Geschlechtsunterschiede: 63 Prozent der Jungen, aber nur 33 Prozent der Mädchen verfügen über eine solche. In den letzten fünf Jahren hat sich die Ausstattung mit Konsolen verdoppelt: 1999 hatten noch 30 Prozent der Jungen und 17 Prozent der Mädchen eine Konsole im eigenen Zimmer.

Ähnliches zeigt sich bei den Nutzungszeiten. Bereits 8- bis 10-Jährige weisen eine tägliche Fernsehnutzungszeit von 197 Minuten auf, Computerspiele werden bereits 65 Minuten genutzt. Aus der Gesamtstichprobe geht hervor, dass die Nutzungszeiten von Computerspielen bei Jungen deutlich höher liegen als bei Mädchen, Jungen spielen insgesamt 72 Minuten, Mädchen 25 Minuten täglich. Die Nutzungszeiten stehen dabei in unmittelbarem Zusammenhang mit der Ausstattung, die Nutzungszeiten liegen deutlich höher, wenn das entsprechende Gerät im eigenen Zimmer vorhanden ist.

Von besonderem Interesse sind die Daten der Studie zum Zusammenhang zwischen Mediennutzung und Schulleistung. Deskriptiv gehen aus den Daten zunächst negative Zusammenhänge zwischen DVD-, Film- und Videonutzung, Musikhören und Schulleistung hervor, die jedoch keine statistische Signifikanz erreichen. Nur für Computerspielen und Lesen werden die Zusammenhänge mit schulischen 
Leistungen statistisch bedeutsam. So berichten gute Schüler (hauptsächlich A und B Noten) über 17 Minuten mehr tägliche Lesezeit als Schüler die C und D Noten als für sich typisch angeben $(p<.05)$. Die schlechteren Schüler spielen hingegen täglich ca. 20 Minuten länger Computerspiele als Schüler mit guten Noten $(p<.05)$ (Roberts et al., 2005).

Für den deutschen Sprachraum ermittelte die PISA-Studie zunächst einen positiven Zusammenhang zwischen der heimischen Verfügbarkeit eines Computers und höheren Kompetenzen der Jugendlichen in Mathematik und im Lesen (OECD, 2001). Die Autoren weisen jedoch darauf hin, dass diese Korrelation mit Vorsicht zu interpretieren sei, da mögliche Einflussvariablen nicht berücksichtigt wurden und keine Aussage über die Wirkungsrichtung getroffen werden kann. In einer ReAnalyse der PISA-Daten durch Fuchs, T. und Wößmann (2004) am Ifo Institute for Economic Research wurde der Zusammenhang zwischen Schulleistung und der Verfügbarkeit und Nutzung von Computern in Schule und im Haushalt eingehender untersucht. Schon bei Kontrolle des familiären Hintergrundes (elterliche Erziehung, Migrationsstatus, sozioökonomischer Status etc.) innerhalb einer multivariaten Regression wird der von PISA noch vorsichtig berichtete positive Zusammenhang zwischen Computerverfügbarkeit zu Hause und Mathematikleistung sowie Lesefähigkeit signifikant negativ, d. h. eine Computerverfügbarkeit zu Hause ging mit schlechteren Leistungen einher. Bei Berücksichtigung weiterer Variablen (Schülereigenschaften, Ressourcen, Schuleigenschaften etc.) haben Schüler mit einem Computer zu Hause um 5.9 Punkte schlechtere Mathematikleistungen ${ }^{5}$ und um 6.5 Punkte schlechtere Lesefertigkeiten ${ }^{5}$ als Schüler ohne Computer. Sind mehr als ein Computer zu Hause vorhanden, sinken die Leistungen in Mathematik um 13.86 Punkte und die Lesefertigkeit um 15.6 Punkte.

In Bezug auf die Verfügbarkeit von Computern an Schulen konnten die Autoren zudem belegen, dass auch die Verfügbarkeit von Computern an Schulen offenbar mit dem Vorhandensein anderer schulischer Ressourcen konfundiert ist (Klassengröße, Erfahrung des Lehrers, Zeit für Hausaufgaben etc.). Werden diese Ressourcen statistisch konstant gehalten, wird der Zusammenhang nicht mehr signifikant. Die Autoren stellen zudem fest, dass der heimische Computer hauptsächlich als Plattform zum Spielen von Computerspielen dient und Schüler vom Lernen ablenkt (Fuchs, T. \& Wößmann, 2004). Die Auswirkung von Computernutzung in der Schule scheint einem umgekehrten U-Kurvenverlauf zu folgen: Kinder, die niemals den Computer oder das Internet in der Schule nutzen, zeigen schlechtere Leistungen als Kinder, die als moderate Nutzer eingestuft werden können. Eine Nutzung mehrmals in der Woche geht aber wiederum mit schlechteren Leistungen einher (Fuchs, T. \& Wößmann, 2004). Diese Ergebnisse überraschen nicht angesichts anderer Studien, die ebenfalls außerhalb spezieller Anwendungskontexte (z. B. Förderung spezifischer Lernschwächen) kaum positive Auswirkungen von Computern im Schulein- 
satz zur Vermittlung schulischer Curricula haben feststellen können (vgl. Angrist \& Lavy, 2002).

Zusammengefasst ergeben die vorgestellten Studien folgendes Bild: Sowohl für das Fernsehen als auch für das Spielen von Computerspielen steht die Medienausstattung von Kindern und Jugendlichen in engem Zusammenhang zu ihren Nutzungszeiten. Hierbei zeigen sich geschlechtstypische Unterschiede im Sinne besserer Ausstattung und höheren Nutzungszeiten bei Jungen. Beide Variablen haben wiederum einen negativen Einfluss auf die Schulleistungen der Kinder und Jugendlichen. Mögliche Erklärungen für diesen Zusammenhang sollen im Folgenden dargestellt werden. 